A

$\begin{array}{cccccccc}\text { tRNA aminoacylation (\%) } & & & & \\ \text { time (min) } & \text { Endo1 } & \text { Endo2 } & \text { Endo3 } & \text { Means Endo } & \text { S.D. Endo } & & \\ 5 & 0,0 & 3,0 & 0,8 & 1,3 & 1,3 & & \\ 10 & 6,7 & 11,9 & 12,1 & 10,2 & 2,5 & \\ 15 & 22,5 & 20,8 & 17,9 & 20,4 & 1,9 & & \\ & & & & & & & \\ 5 & \text { Opt1 } & \text { Opt2 } & \text { Opt3 } & \text { Means Opt } & \text { S.D. Opt } & & \\ 10 & 2,0 & 1,1 & 3,7 & 2,2 & 1,1 & & \\ 15 & 14,2 & 6,2 & 4,7 & 8,3 & 4,2 & & \\ & 22,0 & 13,1 & 16,2 & 17,1 & 3,7 & & \\ 5 & \text { Harm1 } & \text { Harm2 } & \text { Harm3 } & \text { Means Harm } & \text { S.D. Harm } & \text { p-value (Endo) } & \text { p-value (Opt) } \\ 5 & 43,9 & 36,7 & 24,4 & 35,0 & 8,0 & 0,025 & 0,027 \\ 10 & 71,4 & 62,0 & 50,4 & 61,3 & 8,6 & 0,009 & 0,005 \\ 15 & 103,7 & 71,7 & 60,7 & 78,7 & 18,2 & 0,044 & 0,037\end{array}$

B

\begin{tabular}{|c|c|c|c|c|c|c|c|c|c|c|c|c|}
\hline & $\mathrm{Km} 1$ & $\mathrm{Km} 2$ & $\mathrm{Km} 3$ & $\mathrm{Km} 4$ & kcat1 & kcat2 & kcat3 & kcat4 & Means (Km) & S.D. $(\mathrm{Km})$ & Means (kcat) & S.D. (kcat) \\
\hline E. coli $\mathrm{RNNA}^{\mathrm{Tyr}}$ & 0,97 & 0,96 & 0,6 & 1,07 & 8,1 & 7,6 & 13,8 & 18,3 & 0,9 & 0,21 & 12,0 & 5,08 \\
\hline $\begin{array}{l}P f \text {-apitRNA } A^{\mathrm{Tyr}} \\
P f \text {-apitRNA }\end{array}$ & 0,48 & 0,95 & 1 & & 12 & 16 & 20 & & 0,8 & 0,29 & 16,0 & 4,00 \\
\hline \multicolumn{13}{|l|}{ Acceptor stem } \\
\hline G73 & 1,3 & 1,1 & 0,73 & & 16 & 6,9 & 6,5 & & 1,0 & 0,29 & 9,8 & 5,37 \\
\hline $\mathrm{C} 73$ & 0,32 & 0,56 & 0,66 & & 4,4 & 3,6 & 4,4 & & 0,5 & 0,17 & 4,1 & 0,46 \\
\hline U73 & 0,56 & 0,71 & 1,2 & & 3 & 3,1 & 6 & & 0,8 & 0,33 & 4,0 & 1,70 \\
\hline G1-C72 & 1 & 0,3 & 2,2 & & 13 & 10,8 & 7 & & 1,2 & 0,96 & 10,3 & 3,04 \\
\hline C1-G72 & 0,35 & 0,71 & 0,22 & & 5 & 6,5 & 2 & & 0,4 & 0,25 & 4,5 & 2,29 \\
\hline C2-G71/U3-A70 & 0,6 & 0,3 & 0,3 & & 3,6 & 3 & 2,4 & & 0,4 & 0,17 & 3,0 & 0,60 \\
\hline \multicolumn{13}{|c|}{ Anticodon triplet } \\
\hline С34 & 10,8 & 2,45 & 6 & 5,5 & 5,5 & 2,2 & 3 & 2,1 & 6,2 & 3,45 & 3,2 & 1,59 \\
\hline G35 & 0,5 & 0,9 & 0,55 & & 9 & 20,8 & 10 & & 0,7 & 0,22 & 13,3 & 6,54 \\
\hline C35 & 1,9 & 1,85 & 1 & & 3,7 & 6 & 2,8 & & 1,6 & 0,51 & 4,2 & 1,65 \\
\hline A35 & 1,35 & 2,5 & 1,22 & & 5,1 & 3,6 & 4,7 & & 1,7 & 0,70 & 4,5 & 0,78 \\
\hline C36 & 0,4 & 0,9 & 0,4 & & 2,7 & 4,3 & 3,5 & & 0,6 & 0,29 & 3,5 & 0,80 \\
\hline U36 & 0,8 & 1,4 & 0,8 & & 7,3 & 7,8 & 5 & & 1,0 & 0,35 & 6,7 & 1,49 \\
\hline G36 & 1,7 & 1,5 & 1 & & 11 & 11 & 7,1 & & 1,6 & 0,36 & 9,7 & 2,25 \\
\hline $\operatorname{Ser}\left(\mathrm{C}_{35} \mathrm{U}_{36}\right)$ & 1,3 & 0,9 & 1,6 & & 1,73 & 1,1 & 1,1 & & 1,3 & 0,35 & 1,3 & 0,36 \\
\hline \multicolumn{13}{|c|}{ Variable region (Vr) } \\
\hline$\Delta \mathrm{Vr}$ & 1,8 & 0,85 & 2,85 & & 5 & 3,5 & 5,1 & & 1,8 & 1,00 & 4,5 & 0,90 \\
\hline SerVr & 1,4 & 0,6 & 0,42 & 0,8 & 7 & 3 & 4,4 & 8 & 0,8 & 0,43 & 5,6 & 2,30 \\
\hline \multicolumn{13}{|l|}{ D-loop (D-I) } \\
\hline $\mathrm{U} 2 \mathrm{~B}$ & 0,43 & 1 & 1,3 & & 11 & 8,5 & 5 & & 0,9 & 0,44 & 8,2 & 3,01 \\
\hline SerVr+D-1 & 1 & 0,7 & 1,25 & & 10 & 7 & 5,3 & & 1,0 & 0,28 & 7,4 & 2,38 \\
\hline
\end{tabular}


E. coli tRNA ${ }^{\mathrm{Tyr}}$

kcat/KM(1) kcat/KM(2) kcat/KM(3) kcat/KM(4) Means (kcat/Km)

$P f$-apitRNA ${ }^{\mathrm{Ty}}$

$$
\begin{aligned}
& 8,4 \\
& 25,0
\end{aligned}
$$

$\begin{array}{ll}7,9 & 23,0 \\ 16,8 & 20,0\end{array}$

14,1
20,6

1,5

0,197

\section{Acceptor stem}

G73

C73
U73

G1-C72

C1-G72

C2-G71/U3-A70

$\begin{array}{lll}12,3 & 6,3 & 8,9 \\ 13,8 & 6,4 & 6,7\end{array}$

$\begin{array}{ccc}13,8 & 6,4 & 6,7 \\ 5,4 & 4,4 & 5,0\end{array}$

$\begin{array}{rrr}5,4 & 4,4 & 5,0 \\ 13,0 & 36,0 & 3,2\end{array}$

$\begin{array}{ccc}14,3 & 9,2 & 9,1\end{array}$

Anticodon triplet

C34

G35

C35

A35
C36

U36

$\operatorname{Ser}\left(\mathrm{C}_{35} \mathrm{U}_{36}\right)$

$0,5=0,5$

$\begin{array}{ccc}0,5 & 0,9 & 0,5 \\ 18,0 & 23,1 & 18,2\end{array}$

$\begin{array}{ccc}0,5 & 0,9 & 0,5 \\ 18,0 & 23,1 & 18,2 \\ 1,9 & 3,2 & 2,8\end{array}$

$\begin{array}{lll}1,9 & 3,2 & 2,8 \\ 3,8 & 1,4 & 3,9\end{array}$

$\begin{array}{lll}6,8 & 4,8 & 8,8 \\ 9,1 & 5,6 & 6,3\end{array}$

Variable region (Vr)

$\Delta \mathrm{Vr}$

$\begin{array}{cc}2,8 & 4,1 \\ 5 & 5\end{array}$

D-loop (D-I)

U20B

SerVr+D-

25,6
10

8,5
10

4,2

0,4

$\begin{array}{ccc}9,2 & 2,2 & 0,021 \\ 8,9 & 2,3 & 0,026 \\ 4,9 & 4,2 & 0,021 \\ 17,4 & 1,2 & 0,793 \\ 10,8 & 1,9 & 0,033 \\ 8,0 & 2,6 & 0,019\end{array}$

$36,0 \quad 0,014$

$\begin{array}{ll}1,0 & 0,786 \\ 7,7 & 0,015\end{array}$

$\begin{array}{ll}6,8 & 0,011 \\ 3,0 & 0,015\end{array}$

$\begin{array}{ll}6,8 & 0,015 \\ 3,0 & 0,016\end{array}$

$\begin{array}{ll}3,0 & 0,028\end{array}$

$19,1 \quad 0,014$

$7,1 \quad 0,012$

0,013

$\begin{array}{ccc}12,6 & 1,6 & 0,353 \\ 8,1 & 2,1 & 0,047\end{array}$

